RICYDE. Revista Internacional de Ciencias del Deporte doi: $10.5232 /$ ricyde

Rev. int. cienc. deporte

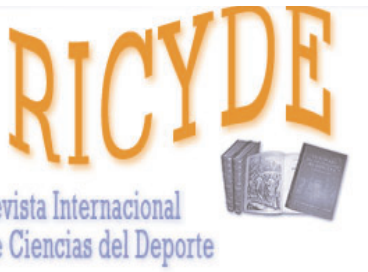

RICYDE. Revista Internacional de Ciencias del Deporte VOLUMEN XI - AÑO XI

Páginas:163-172 ISSN:1885-3137 No 40 - Abril - 2015

\title{
Predicción del motivo salud en el ejercicio físico en centros de fitness Prediction of health reason in physical exercise in fitness centers
}

\author{
Fernanda Borges-Silva1, Ana Prieto-Vaello1, Antonio Alias ${ }^{1}$, y Juan A. Moreno-Murcia ${ }^{2}$
}

1. Universidad Católica San Antonio de Murcia

2. Universidad Miguel Hernández de Elche

\section{Resumen}

El objetivo de este estudio fue comprobar si el apoyo de la autonomía, las necesidades psicológicas básicas y la motivación intrínseca predicen el motivo de práctica hacia la mejora o mantenimiento de la salud. La muestra estuvo compuesta por 602 practicantes de actividades dirigidas con ritmo coreografiados (zumba, aeróbic, step, fight box, batuka, danza contemporánea, flamenco, sevillanas y bailes latinos), a los que se midió el apoyo de autonomía, las necesidades psicológicas básicas, la motivación intrínseca y el motivo salud. Tras el análisis de regresión lineal múltiple por pasos, la salud fue predicha positivamente por el soporte de autonomía del técnico, la satisfacción de los mediadores psicológicos y la motivación intrínseca. Se discuten los resultados en relación al diseño de programas de intervención en busca de estilos de vida más saludables.

Palabras clave: motivación intrínseca; competencias psicológicas básicas; apoyo a la autonomía; clases dirigidas.

\begin{abstract}
The aim of this study was to test if health being the reason of sport practice can be predicted by the support of autonomy, basic psychological needs and intrinsic motivation. A total of 602 practitioners participated in activities aimed at choreographed rhythm (zumba, aerobics, step, box fight, batuka, contemporary dance, flamenco, sevillanas and Latin dances) where the factors support of autonomy, basic psychological needs, intrinsic motivation and health as the reason, were measured. After the stepwise multiple linear regression analysis, the factor health was positively predicted by the technical support of autonomy, the satisfaction of psychological mediators and the intrinsic motivation. The results are discussed in relation to the design of intervention programs in search of healthier life styles.
\end{abstract}

Key words: intrinsic motivation; basic psychological needs; support of autonomy; classes aimed at rhythm. 
Borges-Silva, F.; Prieto-Vaello, A.; Alias, A.; Moreno-Murcia, J. A. (2015). Predicción del motivo salud en el ejercicio físico en centros de fitness. RICYDE. Revista internacional de ciencias del deporte, 40(11), 163-172. http://dx.doi.org/10.5232/ricyde2015.04005

\section{Introducción}

Q egún el postulado de la Teoría de la Autodeterminación (TAD) de Deci y Ryan (1985,

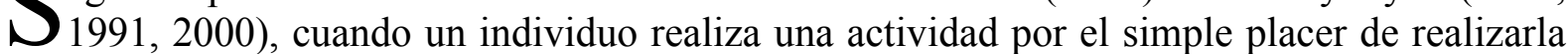
sin ningún incentivo externo (motivación intrínseca), se acercará más a una conducta voluntaria. Vallerand (2007) ha mostrado que poseer una motivación intrínseca para la práctica de ejercicio físico favorece la aparición de patrones conductuales, cognitivos y afectivos más positivos como el placer, el interés, el esfuerzo, la total inmersión en la actividad, la ejecución eficaz o la intención de continuar practicando.

Diversos factores o desencadenantes sociales ejercen sobre la conducta y motivación del individuo (Vallerand, 1997, 2001). En los centros de fítness el técnico responsable por impartir la actividad se convierte en una referencia importante, de tal forma que, cómo se ofrece la información y la interacción con el practicante podría derivar en consecuencias (comportamentales, cognitivas y afectivas) variadas.

En este sentido, uno de los estilos que parecen obtener mejores consecuencias es el apoyo de la autonomía frente al estilo controlador (Torregrosa, Belando, y Moreno-Murcia, 2014; Balaguer, Castillo, y Duda, 2008; y Sarrazin, Vallerand, Guillet, Pelletier, y Cury, 2002). El apoyo a la autonomía por parte del técnico se caracteriza por la posibilidad de elección en las tareas, reduciendo así la necesidad de realizarlas por imposición del mismo y fomentando, por tanto, la iniciativa en la toma de decisiones del practicante (Deci y Ryan, 1985, 1991). Actuar con autonomía significa actuar con un sentido de volición y con la percepción de que se puede elegir (Ryan y Deci, 2000).

Se ha relacionado positivamente el grado en que el practicante percibe el apoyo a la autonomía por parte del técnico deportivo con la satisfacción de las necesidades psicológicas básicas (Álvarez, Balaguer, Castillo, y Duda, 2009). La teoría de la motivación autodeterminada (Deci y Ryan1985, 1991, 2000) establece que para que el practicante experimente mayor motivación intrínseca debe sentir satisfechas las tres necesidades psicológicas básicas de autonomía, competencia y relación con los demás.

Según Vallerand y Rousseau (2001), si las personas son capaces de elegir, sin presiones externas, aquella decisión que les parezca más apropiada (autonomía), realizan acciones con la seguridad de que el resultado sea aquel que esperan o desean (competencia), y sientan que pueden contar con la colaboración y aceptación de las personas que ellos consideran importantes (relación), se producirá un mayor bienestar personal. Con eso, el practicante tendrá la intención de seguir practicando dicha actividad. En este sentido, algunos estudios en el ámbito de la actividad física saludable (Edmunds, Ntoumanis, y Duda, 2006; Wilson y Rodgers, 2004) han revelado que un mayor soporte de autonomía en los entrenamientos, mediado por la satisfacción de las necesidades psicológicas básicas, aumentaría la motivación más autodeterminada.

La búsqueda de estilos de vida activos y saludables es el principal perfil de usuarios de los centros de fitness (González-Serrano, Huéscar, y Moreno-Murcia, 2013; Moreno-Murcia, Borges, Marcos, Sierra, y Huéscar, 2012; Marcos, Borges, Rodríguez, Huéscar, y MorenoMurcia, 2011). Es notorio el crecimiento de la población que frecuenta estos centros atraída por este nuevo estilo de vida. Aun así, el abandono sigue siendo un gran problema en la práctica de ejercicio físico. Por eso, crear conductas que favorezcan la motivación intrínseca sería interesante. Por lo que, analizar las relaciones entre las variables que determinan la práctica podría ayudar a explicar un poco más este contexto de práctica. 
Borges-Silva, F.; Prieto-Vaello, A.; Alias, A.; Moreno-Murcia, J. A. (2015). Predicción del motivo salud en el ejercicio físico en centros de fitness. RICYDE. Revista internacional de ciencias del deporte, 40(11), 163-172. http://dx.doi.org/10.5232/ricyde2015.04005

Preocupados por ello, el objetivo del estudio fue comprobar si el apoyo de la autonomía, las necesidades psicológica básicas y la motivación intrínseca predicen el motivo de práctica salud. En analogía con los estudios revisados, la hipótesis planteada para este estudio fue que el apoyo a la autonomía por parte del técnico deportivo sobre el practicante, la satisfacción de las necesidades psicológicas básicas (autonomía, relación con los demás y competencia) y la motivación intrínseca predirán el motivo de práctica saludable.

\section{Método}

\section{Participantes}

La muestra del estudio estuvo compuesta por 602 practicantes, 237 chicos y 365 chicas, practicantes de actividades dirigidas con ritmo no competitivas (zumba, aeróbic, step, fight box, batuka, danza contemporánea, flamenco, sevillanas y bailes latinos), inscrita en 16 centros deportivos y de baile. Las edades de los participantes oscilaban entre los 18 y 69 años $(M=37.24, D T=10.62)$. El método de selección fue aleatorio.

\section{Instrumentos}

Percepción de apoyo a la autonomía. Se empleó la Escala de Apoyo a la autonomía Percibido en Contextos de Ejercicio (PASSES) de Hagger, Chatzisarantis, Hein, Pihu, Soós, y Karsai (2007), validada al contexto español por Moreno, Parra y González-Cutre (2008). El instrumento encabezado por la sentencia "En mis prácticas de ejercicio físico dirigido...", estaba compuesto por un total de 11 ítems, (e.g., "El monitor/a me facilita con distintas opciones cómo realizar el ejercicio físico"). Las respuestas fueron puntuadas en una escala Likert de 1 (Totalmente en desacuerdo) a 7 (Totalmente de acuerdo). La consistencia interna fue de .87 .

Necesidades psicológicas básicas. Se utilizó la versión en español (Sánchez y Nuñez, 2007) de la Psychological Need Satisfaction in Exercise Scale (PNSE) de Wilson, Rogers, Rodgers, y Wild (2006). El inventario encabezado por la sentencia "En mis prácticas...", estaba compuesto por un total de 18 ítems, seis para evaluar cada una de las necesidades: competencia (e.g., "Tengo confianza para hacer los ejercicios más desafiantes"), autonomía (e.g., "Creo que puedo tomar decisiones respecto a mi programa de ejercicios"), y relación con los demás (e.g., "Me siento cercano a mis compañeros de ejercicios porque ellos saben lo difícil que pueden ser los ejercicios"). Las respuestas fueron recogidas en una escala tipo Likert, cuyo rango de puntuación oscilaba entre 1 (Falso) y 6 (Verdadero). La consistencia interna fue de .89 para la competencia, .85 para la autonomía y .83 para la relación con los demás.

Motivación intrínseca. Se empleó el factor regulación intrínseca de la versión traducida al castellano por Moreno, Cervelló, y Martínez Camacho (2007) del Behavioural Regulation in Exercise Questionnaire (BRSQ) de Lonsdale, Hodge, y Rose (2008). Este factor, encabezado por el enunciado "Participo en este deporte...", estaba compuesto por un total de 4 ítems, (e.g. "Porque es una oportunidad de ser quien soy") que se respondían en una escala Likert de 1 (Nada es verdad) a 7 (Muy verdadero). La consistencia interna fue de .79.

Salud/fitness. Se empleó el factor salud del Cuestionario de contenido de los objetivos en el ejercicio (GCEQ) de Sebire, Standage, y Vansteenkiste (2008), validado al contexto español por Moreno-Murcia, Marcos, y Huéscar (en prensa). Este factor, encabezado por la sentencia "Participo en este deporte...", estaba compuesto por un total de 4 ítems, ("Para ser más resistente a la enfermedad, para aumentar mi nivel de energía, para mejorar mi salud en general y para mejorar mi resistencia"). Las respuestas fueron puntuadas en una escala Likert de 1 (Nada es verdad) a 7 (Muy verdadero). La consistencia interna fue de .84 . 
Borges-Silva, F.; Prieto-Vaello, A.; Alias, A.; Moreno-Murcia, J. A. (2015). Predicción del motivo salud en el ejercicio físico en centros de fitness. RICYDE. Revista internacional de ciencias del deporte, 40(11), 163-172. http://dx.doi.org/10.5232/ricyde2015.04005

\section{Procedimiento}

Una vez establecida la muestra objeto de estudio nos pusimos en contacto con el máximo responsable de los centros deportivos y de baile seleccionados y con los monitores, para informarles de nuestros objetivos y pedirles su colaboración. La administración de los cuestionarios tuvo lugar estando presente el investigador principal, para hacer una breve explicación del objetivo de estudio, informar de cómo cumplimentar los instrumentos y solventar todas las dudas que pudieran surgir durante el proceso, insistiendo en el anonimato de las respuestas y en que se contestara con sinceridad y leyendo todos los ítems. El tiempo requerido para rellenar las escalas fue de aproximadamente 15 minutos, variando ligeramente según la edad del participante. Tanto los centros deportivos y de baile a los que se acudió, como los monitores de las actividades de las clases requeridas y los participantes matriculados en las mismas, participaron voluntariamente en el desarrollo de la investigación.

\section{Análisis de datos}

Se calcularon los estadísticos descriptivos de todas las variables objeto de estudio (medias y desviaciones típicas) y las correlaciones bivariadas. Para conocer la consistencia interna de cada dimensión se utilizó el coeficiente de alfa de Cronbach. Asimismo, se comprobó el poder predictivo del apoyo a la autonomía del técnico deportivo, los mediadores psicológicos y la motivación intrínseca sobre el motivo salud, a través de un análisis de regresión lineal múltiple por pasos. El tratamiento de los datos se realizó con el paquete estadístico SPSS 21.0.

\section{Resultados}

\section{Análisis descriptivos y de correlación}

El apoyo del monitor obtuvo una puntuación de 5.43. El mediador más valorado fue la competencia, seguido de la relación con los demás y la autonomía. La motivación intrínseca presentó un valor medio de 5.50. Los datos revelaron una puntuación en el motivo salud de 5.70. En el análisis de correlación se observó que todos los factores correlacionaron de forma positiva y significativa entre sí (Tabla 1). Es interesante destacar que la relación más baja fue entre los factores autonomía y apoyo a la autonomía. Se puede observar que la autonomía fue el factor de menor puntuación, eso quiere decir que aunque el técnico propiciara autonomía, los sujetos no se sentían autónomos para realizar la actividad.

Tabla 1. Estadísticos Descriptivos y Correlaciones de las Variables de Estudio

\begin{tabular}{|c|c|c|c|c|c|c|c|c|c|c|}
\hline Variables & $M$ & $D T$ & $\mathrm{R}$ & $\alpha$ & 1 & 2 & 3 & 4 & 5 & 6 \\
\hline 1. Apoyo autonomía monitor & 5.43 & 1.22 & $1-7$ & .87 & - & $.40 * *$ & $.19^{* *}$ & $.32 * *$ & $.39 * *$ & $.36^{* *}$ \\
\hline 2. Competencia & 4.73 & .90 & $1-6$ & .89 & - & - & $.48^{* *}$ & $.52 * *$ & $.53^{* *}$ & $.42 * *$ \\
\hline 3. Autonomía & 4.22 & 1.02 & $1-6$ & .85 & - & - & - & $.42 * *$ & $.31 * *$ & $.25^{* *}$ \\
\hline 4. Relación con los demás & 4.28 & .96 & $1-6$ & .83 & - & - & - & - & $.41 * *$ & $.32 * *$ \\
\hline 5. Motivación intrínseca & 5.50 & 1.02 & $1-7$ & .79 & - & - & - & - & - & $.47 * *$ \\
\hline 6. Salud & 5.70 & 1.09 & $1-7$ & .84 & - & - & - & - & - & - \\
\hline
\end{tabular}


Borges-Silva, F.; Prieto-Vaello, A.; Alias, A.; Moreno-Murcia, J. A. (2015). Predicción del motivo salud en el ejercicio físico en centros de fitness. RICYDE. Revista internacional de ciencias del deporte, 40(11), 163-172. http://dx.doi.org/10.5232/ricyde2015.04005

\section{Modelo de regresión lineal}

Para analizar la relación entre las variables estudiadas se realizó un análisis de regresión lineal múltiple por pasos. Se utilizó el método de pasos sucesivos (Stepwise) en la introducción de las variables independientes. En cada paso se introdujo la variable independiente. Las variables ya introducidas en la ecuación de regresión se eliminaban de ella si su probabilidad para F llegaba a ser suficientemente grande. El método terminó cuando ya no había más variables candidatas a ser incluidas o eliminadas. El criterio para la introducción por pasos fue el presentado por Vallerand (2001) en su modelo jerárquico de motivación intrínseca y extrínseca, donde se partió desde los desencadenantes, se pasó a los mediadores y así sucesivamente hasta llegar las posibles consecuencias que se quieren que se predigan. Se utilizó como variable dependiente el motivo salud y como variables predictoras fueron utilizadas el apoyo a la autonomía, las necesidades psicológicas básicas y la motivación intrínseca. En el primer paso del análisis de regresión lineal el apoyo a la autonomía del técnico deportivo predijo positivamente el motivo salud con un $13 \%$ de varianza explicada. En el segundo paso, se introdujo las necesidades psicológicas que predijeron positivamente el motivo salud junto al soporte de autonomía con un $23 \%$ de varianza explicada. En el tercer paso el apoyo a la autonomía del técnico deportivo, las necesidades psicológicas y la motivación intrínseca, predijeron positivamente el motivo salud con un $28 \%$ de varianza explicada (Tabla 2).

Tabla 2. Análisis de Regresión Lineal de Predicción del Motivo Salud a Través del Apoyo a la Autonomía del Técnico Deportivo, las Necesidades Psicológicas y la Motivación Intrínseca

\begin{tabular}{|c|c|c|c|c|}
\hline & $B$ & $S E B$ & $\beta$ & $\Delta R^{2}$ \\
\hline Primer paso & 3.93 & .18 & & $.13^{* *}$ \\
\hline Apoyo autonomía monitor & 32 & .03 & $.36^{*}$ & \\
\hline Segundo paso & 2.46 & .24 & & $23^{* *}$ \\
\hline Apoyo autonomía monitor & .19 & .03 & $.22 *$ & \\
\hline Autonomía & .04 & .04 & $.04 *$ & \\
\hline Competencia & .32 & .05 & $.26^{*}$ & \\
\hline Relación con los demás & .10 & .04 & $.09 *$ & \\
\hline Tercer paso & 1.89 & .25 & & $.28 * *$ \\
\hline Apoyo autonomía monitor & .14 & .03 & $.16^{*}$ & \\
\hline Autonomía & .03 & .04 & $.03 *$ & \\
\hline Competencia & .19 & .05 & $.16^{*}$ & \\
\hline Relación con los demás & .05 & .04 & $.04 *$ & \\
\hline Motivación intrínseca & .30 & .04 & $.28 * *$ & \\
\hline
\end{tabular}

Nota: ${ }^{*} p<.05 ; * * p<.01$. 
Borges-Silva, F.; Prieto-Vaello, A.; Alias, A.; Moreno-Murcia, J. A. (2015). Predicción del motivo salud en el ejercicio físico en centros de fitness. RICYDE. Revista internacional de ciencias del deporte, 40(11), 163-172. http://dx.doi.org/10.5232/ricyde2015.04005

\section{Discusión}

El objetivo del trabajo fue comprobar la predicción del motivo salud de práctica a través del soporte de autonomía del técnico deportivo, las necesidades psicológicas básicas y la motivación intrínseca. La hipótesis planteada fue confirmada, el soporte de autonomía del técnico deportivo, la satisfacción de las necesidades psicológicas básicas (autonomía, relación con los demás y competencia) y la motivación intrínseca han predicho positivamente el motivo salud. Aunque existen estudios (Moreno y col., 2009; Moreno y col., 2008) que han encontrado que la satisfacción de las necesidades psicológicas básicas y la motivación intrínseca se relacionan positivamente con el motivo salud en programas de ejercicio físico no competitivo, este trabajo resulta pionero al analizar el soporte de autonomía como desencadenante social en el ámbito de actividades dirigidas con ritmo coreografiados en centros de fitness.

Los participantes otorgan un gran valor al motivo salud para realizar ejercicio físico, corroborando los hallazgos de investigaciones anteriores (Castillo y Balaguer, 2001; García Fernando, 2006; Navarro, González-Cutre, Marcos, Borges, Hernández, A., Vera, y Moreno, 2008). Además, presenta una relación positiva con la autonomía que el técnico deportivo reporta sobre el practicante, las necesidades psicológicas básicas (autonomía, relación con los demás y competencia) y la motivación intrínseca. Esta relación positiva entre la percepción del apoyo a la autonomía por parte del técnico deportivo y las relaciones sociales percibidas también ha sido encontrada en otros estudios anteriores, en muestras de deportistas (Balaguer, Castillo, y Duda, 2005 y Vallerand y Losier, 1999, Belando, Ferriz-Morell, y Moreno-Murcia, 2012) y en el ámbito del fitness (Moreno y col., 2010). Por lo que generar un clima que tenga en cuenta al practicante, permitiendo que este participe en el proceso de toma de decisiones y valorando y teniendo en cuenta su opinión, puede ser determinante en su motivación hacia la actividad. Es el técnico deportivo el responsable de intentar generar este clima para que el practicante participe en la actividad a lo largo del tiempo (Chatzisarantis, Hagger, y Brickell, 2008).

Las relaciones encontradas entre la satisfacción de las necesidades psicológicas básicas y la motivación intrínseca se ajustan a los postulados de la teoría de la autodeterminación y van en la línea de los resultados de diferentes trabajos realizados en entornos físico-deportivos (Edmunds, Ntoumanis, y Duda, 2006; Sarrazin, Vallerand, Guillet, Pelletier, y Cury, 2002; Standage, Duda y Ntoumanis, 2006). En concreto, según Moreno y col., (2010), es más probable que la persona alcance la motivación intrínseca (acción promovida por el disfrute obtenido durante la actividad) en aquellas situaciones en las que se permita la autonomía, se garantice la consecución de los objetivos establecidos (competencia) y se fomente la cohesión del grupo (relación con los demás).

Las personas que realizan ejercicio físico para mejorar la salud parece ser que también se siente motivadas intrínsecamente, es decir, aunque su objetivo sea practicar ejercicio físico para la mejora de la salud (motivación extrínseca), también realizan ejercicios por placer en la práctica. Eso puede ser que garantice que una conducta duradera al largo del tiempo.

\section{Conclusiones}

Como conclusión de este trabajo, se indica que el soporte de autonomía generado por el técnico deportivo, así como, la satisfacción de sus necesidades psicológicas básicas y la motivación intrínseca predicen positivamente el motivo de práctica saludable. Esto podría ser concluyente sobre la continuidad en un programa de ejercicio físico (Decy y Ryan, 1985, 1991, 2000). Los resultados obtenidos en coherencia con los estudios considerados, podrían 
Borges-Silva, F.; Prieto-Vaello, A.; Alias, A.; Moreno-Murcia, J. A. (2015). Predicción del motivo salud en el ejercicio físico en centros de fitness. RICYDE. Revista internacional de ciencias del deporte, 40(11), 163-172. http://dx.doi.org/10.5232/ricyde2015.04005

contribuir como guía para la planificación de programas de ejercicio físico. Este modelo predictivo puede suponer un punto de apoyo para el diseño de los programas de intervención de las actividades dirigidas con ritmo coreografiadas, en busca de estilos de vida más saludables. Así mismo, podrían contribuir como guía para la planificación de programas de ejercicio físico bajo metodologías que fomenten la autonomía de los practicantes (tareas abiertas, fomento de la iniciativa, elección de actividades, etc.) ya que parecen relacionarse con una consecución de las necesidades psicológicas básicas (Álvarez, Balaguer, Castillo, y Duda, 2009) así como una mayor motivación intrínseca (Balaguer y col., 2008). En este sentido, establecer estrategias motivacionales para conseguir conductas más adaptativas al ejercicio físico que favorezca la autonomía, la satisfacción de las necesidades psicológicas básicas, puede que posibilite que el practicante esté motivado intrínsecamente.

\section{Limitaciones del estudio}

Respecto a las limitaciones del estudio, cabe destacar que la metodología utilizada ha sido de tipo correlacional. Por lo que sería necesario emplear una metodología experimental pues, aunque las variables manifiesten una relación importante, no implica necesariamente que haya causa-efecto entre ellas (Quinata, 1989). Además, los datos no pueden ser extrapolados más allá de los rangos observados en la muestra del estudio. En este sentido, sería interesante que futuros trabajos corroboren los resultados obtenidos con muestras más amplias, incluyendo el estudio de perfiles motivacionales (Wang y Biddle, 2001), y sobre todo, teniendo en cuenta un mayor número de variables sociodemográficas que la investigación viene considerando influyentes a la hora de practicar actividades físico-deportivas, como la edad de los participantes, el sexo o la práctica en el entorno familiar (Hassandra, Goudas y Chroni, 2003; Moreno y col., 2006), a través de un programa activo de intervención bajo un seguimiento longitudinal.

\section{Referencias}

Álvarez, M. S.; Balaguer, I.; Castillo, I., \& Duda, J. L. (2009). Coach autonomy support and quality of sport engagement in young soccer players. The Spanish Journal of Psychology, 12(1), 138-148.

http://dx.doi.org/10.1017/S1138741600001554

Balaguer, I.; Castillo, I.; Álvarez, M., \& Duda, J. L. (2005). Importance of social context in the prediction of self-determination and well-being in athletes of different level. En Symposium on "Motivation in Sport and Physical Activity". 9th European Congress of Psychology. Granada: Universidad de Granada.

Balaguer, I.; Castillo, I., y Duda, J. L. (2008). Apoyo a la autonomía, satisfacción de las necesidades, motivación y bienestar en deportistas de competición: un análisis de la Teoría de la autodeterminación. Revista de Psicología del Deporte, 17(1), 123-139.

Belando, N.; Ferriz-Morell, R. y Moreno-Murcia, J. A. (2012). Propuesta de un modelo para la mejora personal y social a través de la promoción de la responsabilidad en la actividad físico-deportiva. RICYDE. Revista Internacional de Ciencias del Deporte, $8(29), 202-222$.

http://dx.doi.org/10.5232/ricyde2012.02902

Castillo, I., y Balaguer, I. (2001). Dimensiones de los motivos de práctica deportiva de los adolescentes valencianos escolarizados. Apunts: Educación Física y Deportes, 63, 22-29.

Chatzisarantis, N.; Hagger, M. \& Brickell, T. (2008). Using the construct of perceptive autonomy support to understand social influence within the theory of planned behavior. Psychology of Sport and Exercise, 9, 27-44.

http://dx.doi.org/10.1016/j.psychsport.2006.12.003 
Borges-Silva, F.; Prieto-Vaello, A.; Alias, A.; Moreno-Murcia, J. A. (2015). Predicción del motivo salud en el ejercicio físico en centros de fitness. RICYDE. Revista internacional de ciencias del deporte, 40(11), 163-172. http://dx.doi.org/10.5232/ricyde2015.04005

Deci, E. L., \& Ryan, R. M. (1985). Intrinsic motivation and self-determination in human behavior. Nueva York: Plenum.

http://dx.doi.org/10.1007/978-1-4899-2271-7

Deci, E. L., y Ryan, R. M. (1991). Un enfoque motivacional para auto: La integración de la personalidad. En R. Dienstbier (Ed.), Nebraska simposio sobre la motivación: Vol. 38. Perspectivas sobre la motivación (pp. 237-288). Lincoln, NE: University of Nebraska Press.

Deci. E. L., \& Ryan, R. M. (2000). The "what" and "why" of goal pursuits: Human needs and the self-determination of behavior. Psychological Inquiry, 11, 227-268. http://dx.doi.org/10.1207/S15327965PLI1104_01

Edmunds, J. M.; Ntoumanis, N., \& Duda, J. L. (2006). A Test of Self-Determination Theory in the Exercise Domain. Journal of Applied Social Psychology, 36(9), 22402265.

http://dx.doi.org/10.1111/j.0021-9029.2006.00102.x

García-Ferrando, M. (2006). Posmodernidad y deporte: Entre la individualización y la masificación. Encuesta sobre hábitos deportivos de los españoles 2005. Madrid: CSD y CIS.

González-Serrano, G.; Huéscar, E., y Moreno-Murcia, J. A. (2013). Satisfacción con la vida y ejercicio físico. Motricidad. European Journal of Human Movement, 30, 131151.

Hagger, M. S.; Chatzisarantis, N. D.; Hein, V.; Pihu, M.; Soós, I., \& Karsai, I. (2007). The perceived autonomy support scale for exercise settings (PASSES): development, validity, and cross-cultural invariance in young people. Psychology of Sport and Exercise, 8(5), 632-653.

http://dx.doi.org/10.1016/j.psychsport.2006.09.001

Lonsdale, C.; Hodge, K., \& Rose, E. A. (2008). The development of the Behavioral Regulation in Sport Questionnaire (BRSQ): Instrument development and initial validity evidence. Journal of Sport and Exercise Psychology, 30, 323-355.

Marcos, P. J.; Borges, F.; Rodríguez, A.; Huéscar, E., y Moreno-Murcia, J. A. (2011). Indicios de cambio en los motivos de práctica físico-deportiva según el sexo y la edad. Apuntes de Psicología, 29, 123-132.

Moreno, J. A.; Borges, F.; Marcos, P. J.; Sierra, A. C., y Huéscar, E. (2012). Motivación, frecuencia y tipo de actividad en practicantes de ejercicio físico. Revista Internacional de Medicina y Ciencias de la Actividad Física y el Deporte, 12(48), 649-662.

Moreno-Murcia, J. A.; Cervelló, E.; Borges, F., y Conte, L. (2009). El interés en la opinión del practicante de ejercicio físico tan importante papel en la predicción de la aptitud / de salud razón. Fitness and Performance Journal, 8(4), 247-253. http://dx.doi.org/10.3900/fpj.8.4.247.s

Moreno, J. A.; Cervelló, E.; Marcos, P., y Martín, E. (2010). Importancia de la valoración del comportamiento autónomo del practicante para el disfrute en programas de ejercicio físico acuático. Cuadernos de Psicología del Deporte, 10(1), 57-70.

Moreno, J. A.; Cervelló, E., y Martínez Camacho, A. (2007). Validación de la Escala de Medida de los Motivos para la Actividad Física-Revisada en españoles: Diferencias por motivos de participación. Anales de Psicología, 23, 167-176.

Moreno-Murcia, J. A.; Cervelló-Gimeno, E.; Martínez-Galindo, M. C., y Moreno R. (2013). Validación de la Escala de Creencias Implícitas de habilidad (CNAAQ-2) al contexto español. Diferencias según la práctica físico-deportiva. RICYDE. Revista Internacional de Ciencias del Deporte, 9(32), 100-113.

http://dx.doi.org/10.5232/ricyde2013.03201 
Borges-Silva, F.; Prieto-Vaello, A.; Alias, A.; Moreno-Murcia, J. A. (2015). Predicción del motivo salud en el ejercicio físico en centros de fitness. RICYDE. Revista internacional de ciencias del deporte, 40(11), 163-172. http://dx.doi.org/10.5232/ricyde2015.04005

Moreno, J. A.; Conte, L.; Borges, F., y González-Cutre, D. (2008). Necesidades psicológicas básicas, motivación intrínseca y propensión a la experiencia autotélica en el ejercicio físico. Revista Mexicana de Psicología, 25(2), 305-312.

Moreno-Murcia, J. A.; Conde, C., y Sáenz-López, P. (2012). Importancia del apoyo de autonomía en la figura del docente de educación física. Tándem. Didáctica de la Educación Física, 40, 18-27.

Moreno, J. A.; González-Cutre, D.; Martín-Albo, J., \& Cervelló, E. (2010). Motivation and performance in physical education: an experimental test. Journal of Sports Science and Medicine, 9, 79-85.

Moreno-Murcia, J. A.; Marcos, P., y Huéscar, E. (en prensa). Motivos de práctica físicodeportiva en mujeres: Diferencias entre practicantes y no practicantes. Cultura y Educación.

Moreno, J. A.; Parra, N., y González-Cutre, D. (2008). Influencia del apoyo a la autonomía, las metas sociales y la relación con los demás sobre la desmotivación en educación física. Psicothema, 20(4), 636-641.

Navarro, N.; González-Cutre, D.; Marcos, P. J.; Borges, F.; Hernández, A., Vera, J. A., y Moreno, J. A. (2008). Perfiles motivacionales en la actividad física saludable: un estudio desde la perspectiva de la teoría de la autodeterminación. En Actas del XI Congreso Nacional, XI Andaluz y III Iberoamericano de Psicología de la Actividad Física y del Deporte. Sevilla: Universidad Pablo de Olavide.

Ryan, R. M., \& Deci, E. L. (2000). Self-determination theory and the facilitation of intrinsic motivation, social development, and wellbeing. American Psychologist, 55, 68-78.

http://dx.doi.org/10.1037/0003-066X.55.1.68

Sánchez, J. M., y Núñez, J. L. (2007). Análisis preliminar de las propiedades psicométricas de la versión española de la Escala de Necesidades Psicológicas Básicas en el Ejercicio Físico. Revista Iberoamericana de Psicología del Ejercicio y el Deporte, 2(2), 83-92.

Sarrazin, P.; Vallerand, R.; Guillet, E.; Pelletier, L., \& Cury, F. (2002). Motivation and dropout in female handballers: A 21-month prospective study. European Journal of Social Psychology, 32, 395-418.

http://dx.doi.org/10.1037/0003-066X.55.1.68

Sebire, S. J.; Standage, M., \& Vansteenkiste, M. (2008). Development and validation of the goal content for exercise questionarire. Journal of Sport and Exercise Psychology, 30, 353-377.

Standage, M.; Duda, J. L., \& Ntoumanis, N. (2006). Students' motivational processes and their relationship to teacher ratings in school physical education: A selfdetermination theory approach. Trimestral de Investigación para el Ejercicio y el Deporte, 77, 100-110.

Torregrosa, D.; Belando, N., y Moreno-Murcia, J. A. (2014). Predicción de la satisfacción con la vida en practicantes de ejercicio físico saludable. Cuadernos de Psicología del Deporte, 14, 117-122.

http://dx.doi.org/10.4321/S1578-84232014000100014

Vallerand, R. J. (1997). Toward a hierarchical model of intrinsic and extrinsic motivation. En M. P. Zanna (Ed.), Advances in experimental social psychology (pp. 271-360). Academic Press: New York.

Vallerand, R. J. (2001). A hierarchical model of intrinsic and extrinsic motivation in sport andexercise. En G. C. Roberts (Ed.), Advances in motivation in sport and exercise (pp. 263-319). Champaign, IL: Human Kinetics. 
Borges-Silva, F.; Prieto-Vaello, A.; Alias, A.; Moreno-Murcia, J. A. (2015). Predicción del motivo salud en el ejercicio físico en centros de fitness. RICYDE. Revista internacional de ciencias del deporte, 40(11), 163-172. http://dx.doi.org/10.5232/ricyde2015.04005

Vallerand, R. J. (2007). Intrinsic and extrinsic motivation in sport and physicalactivity. A review and a look at the future. En G. Tenenbaum y R. C. Eklund (Eds.), Handbook of sportpsychology ( $3^{a}$ ed., pp. 59-83). Nueva York: John Wiley.

Vallerand, R. J., y Losier, G. F. (1999). Un análisis integral de la motivación intrínseca y extrínseca en el deporte. Diario de Psicología del Deporte, 11, 142-169.

Vallerand, R. J., \& Rousseau, F. L. (2001). Intrinsic and extrinsic motivation in sport and exercise: A review using the hierarchical model of intrinsic and extrinsic motivation. En R. N. Singer, H. A. Hausenblas y C. M. Janelle (Eds.), Handbook of Sport Psychology ( $2^{\mathrm{a}}$ ed., pp. 389-416). New York: John Wiley and Sons.

Wilson, P. M., y Rodgers, W. M. (2004). The relationship between perceived autonomy support, exercise regulations and behavioral intentions in women.Psychology of Sport And Exercise, 5(3), 229-242. http://dx.doi.org/10.1016/S1469-0292(03)00003-7

Wilson, P. M.; Rogers, W. T.; Rodgers, M., \& Wild, T. C. (2006). The psychological Need Satisfaction in exercise scale. Journal of Sport and Exercise Psychology, 28, 231-25. 